\title{
Detecting and managing hypertensive disorders in pregnancy: a cross-sectional analysis of the quality of antenatal care in Nigeria
}

\author{
Angela Salomon ${ }^{*^{*}}$, Salisu Ishaku ${ }^{2}$, Karen R. Kirk ${ }^{3}$ and Charlotte E. Warren ${ }^{4}$
}

\begin{abstract}
Background: Nigeria has one of the highest rates of maternal mortality in the world (576/100,000 births), with a significant proportion of death attributed to hypertensive disorders in pregnancy (HDPs). High quality antenatal care (ANC) plays a crucial role in early detection and management of HDPs. We conducted an assessment of quality of antenatal care, and its capacity to detect and manage HDPs, in two tiers of Nigerian facilities, with the aim of describing the state of service delivery and identifying the most urgent gaps.

Methods: Quality of antenatal care was assessed and compared between primary healthcare centers (PHCs) $(n=56)$ and hospitals (secondary + tertiary facilities, $n=39$ ) in seven states of Nigeria. A cross-sectional design captured quality of care using facility inventory checklists, semi-structured interviews with healthcare providers and clients, and observations of ANC consultations. A quality of care framework and scoring system was established based on aspects of structure, process, and outcome. Average scores were compared using independent sample t-tests and measures of effect were assessed by multivariate linear regression.

Results: All domains of quality except provider interpersonal skills scored below 55\%. The lowest overall scores were observed in provider knowledge (49.9\%) and provider technical skill (47.7\%). PHCs performed significantly worse than hospitals in all elements of quality except for provider interpersonal skills. Provider knowledge was significantly associated with their level of designation (i.e., obstetrician vs. other providers).

Conclusions: In order to provide high quality care, ANC in Nigeria must experience massive improvements to inventory, infrastructure and provider knowledge and training. In particular, ANC programs in PHCs must be revitalized to minimize the disparity in quality of care provided between PHCs and hospitals. The relatively low quality of care observed may be contributing to Nigeria's high rate of maternal mortality and burden of disease attributed to HDPs.
\end{abstract}

Keywords: Antenatal care - maternal health - quality of care - health systems, Hypertensive disorders in pregnancy primary health care

\footnotetext{
* Correspondence: angela.salomon94@gmail.com

${ }^{1}$ Dalla Lana School of Public Health, Toronto, Canada

Full list of author information is available at the end of the article
}

(c) The Author(s). 2019 Open Access This article is distributed under the terms of the Creative Commons Attribution 4.0 International License (http://creativecommons.org/licenses/by/4.0/), which permits unrestricted use, distribution, and reproduction in any medium, provided you give appropriate credit to the original author(s) and the source, provide a link to the Creative Commons license, and indicate if changes were made. The Creative Commons Public Domain Dedication waiver (http://creativecommons.org/publicdomain/zero/1.0/) applies to the data made available in this article, unless otherwise stated. 


\section{Background}

Antenatal care (ANC) describes the services offered to pregnant women, including health promotion and communication, screening and diagnosis, disease prevention, and emotional and psychological support. Quality, evidencebased ANC plays a crucial role in improving the lives of pregnant women, and in setting the groundwork for healthy motherhood and infant development [1]. As of 2015, hypertensive disorders in pregnancy (HDPs), including pre-eclampsia and eclampsia, were the greatest cause of facility-based maternal mortality (29.0\%) in Nigeria [2]. Broadly speaking, HDPs are characterized by elevated blood pressure, proteinuria, and many hematologic, hepatic, neurologic and renal changes that can result in adverse maternal and neonatal outcomes including intrauterine growth restriction, oligohydramnios (low amniotic fluid), placental abruption, and fetal death [3]. Because HDPs tend to have easily detectable clinical parameters [4], increasing utilization of quality ANC is essential to reducing maternal mortality caused by this class of disease.

Globally, an estimated $81 \%$ of pregnant women attend at least one antenatal care visit, while only $56 \%$ attend at least four [5]. In regions of sub-Saharan Africa, this number is further reduced [6], and in Nigeria specifically, only half of pregnant women attend at least four visits, ranging from $30 \%$ in north-western states to $87 \%$ in the southwest $[7,8]$. While increasing access to ANC services is important, it is also critical that the services received are of good quality. Quality of Care (QOC), as described by Donabedian [9], depends on three components: structure (adequacy of physical environment and systems), process (components of care delivered), and outcomes (satisfaction/status of clients) $[9,10]$. That is to say, facilities should be well stocked with essential commodities, services should be provided by competent healthcare workers, and clients should leave well-informed, satisfied, and respected.

In Nigeria, primary healthcare centers (PHCs) are the first points of contact for many pregnant women, especially for the majority of rural-dwellers; however, service readiness in PHCs is often sub-standard compared to hospital counterparts $[11,12]$. Recently, a landscape analysis of pre-eclampsia and eclampsia in Nigeria [13] showed limited capacity at PHCs for providing even the most basic emergency obstetric and neonatal care. Previous research has explored factors affecting quality of ANC in Nigeria but has been restricted to private health facilities and small geographic areas and has used a limited scope of quality of care [14-16]. This paper explores the quality of ANC in seven states across the six geopolitical zones of Nigeria using a well-established framework for assessing QOC, with a focus on its capacity to detect and manage hypertensive disorders in pregnancy. Furthermore, it examines disparities in the quality of ANC provided between PHCs and hospitals.

\section{Methods}

\section{Study participants}

Following discussion with the Nigerian Federal Ministry of Health and the donor, seven states covering the country's six geo-political zones were chosen as study sites Sokoto, Bauchi, Katsina, Kogi, Cross River, Ebonyi, and Ondo (Fig. 1). In consultation with the states' Ministries of Health, ninety-five public facilities with ANC units were purposively selected from within these states to represent diversity in facility type ( $\mathrm{PHCs}=56$, secondary/ tertiary hospitals $=39$ ). Following an inventory assessment at 95 selected facilities, researchers conducted service provider interviews $(n=200)$ from a subset of 93 facilities; 2 facilities were excluded as no willing providers were present on the day of the inventory assessment. Researchers also observed 135 ANC consultations and conducted 135 client exit interviews from a subset of 26 facilities. The subset of facilities used for consultation observations and client interviews was chosen using convenience sampling, based on the location of the facility (considering difficult terrains), as well as the functionality of the facility (facility operating hours), while ensuring a representative sample across facility level and region.

\section{Quality of Care framework}

The framework used for measuring quality of ANC was adapted from Warren et al. [17] and included many of the essential elements of antenatal care as informed by WHO guidelines [1]. It was further tailored to include indicators specifically pertaining to the capacity of the ANC to detect and manage HDPs.

The framework incorporated three elements of QOC as described by Donabedian [9], Bruce [18], and Hulton [19]: structure, process, and outcome. Structure encompassed factors related to inventory and infrastructure (facility guidelines, equipment, supplies, drugs, referral mechanisms, etc.), as well as human resources (provider knowledge and training). Process encompassed the range of services provided, information and documentation shared with the client, the practitioner's competency in history taking and physical examinations, and their interpersonal skills. Outcome encompassed client experiences, satisfaction, and health comprehension. Table 1 shows the indicators used to create structure, process, and outcome scores. Scores were calculated as the average cumulative number of points received by each unit of observation (facility, provider, ANC consultation, or client interview). All scores are reported as a percent of the total possible score.

\section{Data collection}

This paper uses cross sectional data collected during a landscape analysis that assessed the health facility and provider capacity to prevent, detect, and manage 




Fig. 1 Map of study sites. Data was collected from 95 participating hospitals in 7 states across the 6 geopolitical zones of Nigeria. Geopolitical zones are divided based on similarity in culture, ethnic group, and common history. Including all hospitals from all geopolitical zones helps to ensure representative national results. Rights to this image are owned by the author

preeclampsia and eclampsia in seven states in Nigeria [13]. Data collection occurred over a three-month period, from June-August 2015.

\section{Structure}

Inventory and infrastructure $(n=95)$ were assessed with a facility inventory checklist for a maximum score of 38 points. Provider knowledge, competence, and training was assessed using semi-structured interviews with the ANC service providers $(n=200)$ for a maximum score of 66 points (Table 1 ).

\section{Process}

Client-provider interactions $(n=135)$ were observed and documented using a standardized checklist to assess the provider's technical skills for a maximum score out of 37, and the provider's interpersonal skills for a maximum score out of 11 (Table 1).

\section{Outcome}

Following their ANC visit, researchers conducted semistructured exit interviews with clients $(n=135)$ to assess their perception, satisfaction, and knowledge of services received, for a maximum score out of 16 (Table 1 ).

\section{Data analysis}

We compared mean scores obtained from PHCs and hospitals in each QOC category using one-tailed independent sample t-tests $\left(\mathrm{H}_{0}\right.$ : secondary facility $=$ PHCs, $\mathrm{H}_{\mathrm{A}}$ : secondary facility $>\mathrm{PHC}$ ); variances were assumed unequal if Levene's Test for Equality was significant. For all categorical variables, significant differences between facility type were determined using the Pearson chi-square test; Fisher's Exact test was used in cases where individual cell counts were below 5 .

We then conducted two multivariate linear regression models to identify independent variables associated with provider knowledge and outcome scores. Variables in model 1 included facility level, provider age, gender, and designation; variables in model 2 included facility level, client age, education, and socio-economic status (SES). Clustering within facility was adjusted for using a robust estimate of variance (sandwich estimator).

We developed a single variable for SES through principal component analysis of indicators pertaining to wealth, household facilities, and assets (including vehicles, land, and livestock owned). We classified SES as either low, medium or high based on whether the individual fell into the bottom, middle, or top tertile [20].

A significance threshold of $p=0.05$ was used for all analyses. Individual observations with $>50 \%$ of data missing were excluded from analysis. Where appropriate, means (with standard deviations), coefficients (with 95\% confidence intervals), and $p$-values are reported. Data were stored, cleaned, and analyzed using STATA v $15.1[21]$ 







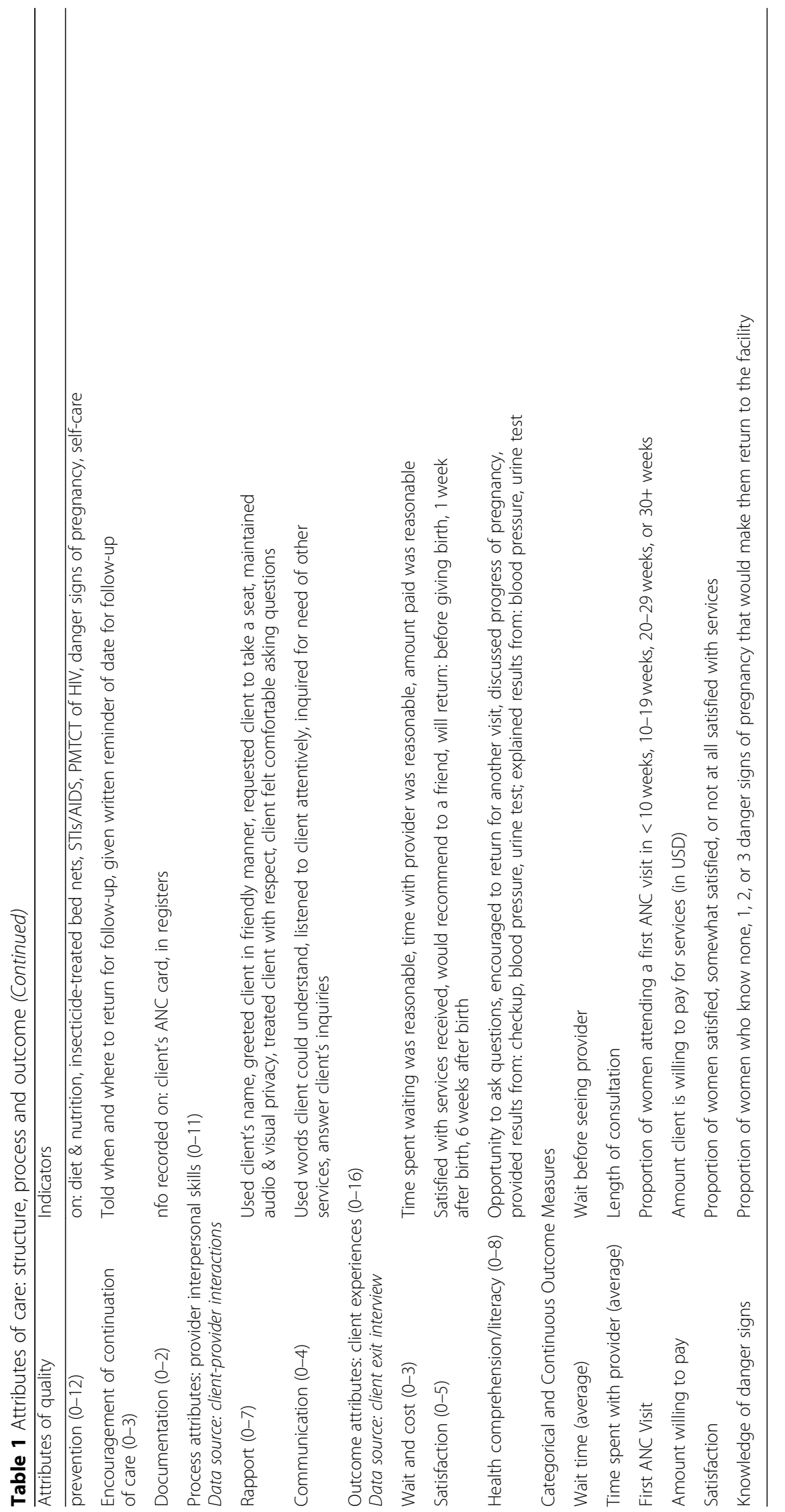




\section{Results}

Figure 2 shows an overview of the scores received in each of the five categories by all facilities, PHCs, and Hospitals.

\section{Descriptive characteristics}

Client and provider characteristics are described in Table 2. The average client was 27.78 years of age, with those visiting PHCs being significantly younger than those visiting hospitals (24.9 vs. 28.3 years; $p=0.001$ ). Most women had completed at least secondary level of schooling (30.1\%), and the level of education attained was significantly associated with facility type, with a much higher percentage of women at hospitals having attended postsecondary school $(p<0.001)$. Those identifying as Muslim were approximately three times more likely to attend a PHC than a hospital facility $(\mathrm{p}=0.001)$, likely because the majority of PHCs sampled were in Northern states, which are predominantly Muslim. There were no significant differences between PHCs and hospitals in the client's SES, marital status, or spending decision habits.

The mean age of providers (39.1 years) was similar at both PHCs and hospitals, and the majority were female (81.6\%). In PHCs, most providers were Community Health Extension Workers (CHEWs) (64.8\%), followed by nurse/midwives (18.1\%); this was significantly different from hospitals, where the majority of provides were nurses/midwives (66.7\%), followed by general practitioners $(15.6 \%)(p<0.001)$.

\section{Structural attributes of care: inventory/infrastructure}

Table 3 shows the structural attributes related to facility inventory and infrastructure. Out of a total of 38 points, PHCs scored significantly lower than hospitals (47.5\% vs. $60.0 \%, \mathrm{p}<0.001)$. PHCs also scored significantly lower in domains of general infrastructure (40.8\% vs. $54.3 \% ; p=0.0116)$, general equipment $(54.2 \%$ vs. $64.6 \%, p=0.0304)$, referral mechanisms $(54.9 \%$ vs. $67.3 \% ; p=0.0161)$ and capacity for HDP specific services $(38.8 \%$ vs. $68.6 \% ; p<0.0001)$. There were no significant differences between facility type in scores for data collection tools (90.5\%), HDP guidelines (33.9\%), or availability of drugs associated with HDPs (38.5\%).

\section{Structural attributes of care: provider knowledge/training}

Table 3 shows the structural attributes related to provider knowledge and training. Out of a total of 66 points, providers in PHCs scored significantly lower than those in hospitals $(46.0 \%$ vs. $54.1 \%, p=0.002)$. PHCs scored lower than hospitals in additional facets of provider knowledge including signs and symptoms of HDPs $(60.1 \%$ vs. $67.8 \%$; $p=0.019)$, how to manage severe preeclampsia $(49.2 \%$ vs. $59.0 \% ; p=0.032)$ and eclampsia (49.7\% vs. $65.2 \%$ ), correctly diagnosing HDPs (58.0\% vs. $73.6 \% ; p=0.000)$, correct use of magnesium sulfate (15.9\% vs. $31.1 \%, \mathrm{p}=0.000)$, and use of antihypertensive drugs to manage mild HDP (13.6\% vs. $23.5 \%, p=0.001)$.

The difference observed in overall provider knowledge between PHCs and hospital facilities was no longer significant after regression adjustment for provider demographic variables including age, gender, type of provider, and length of time working at facility (Fig. 3). However, overall provider knowledge was significantly associated with provider type; using maternal health specialists as

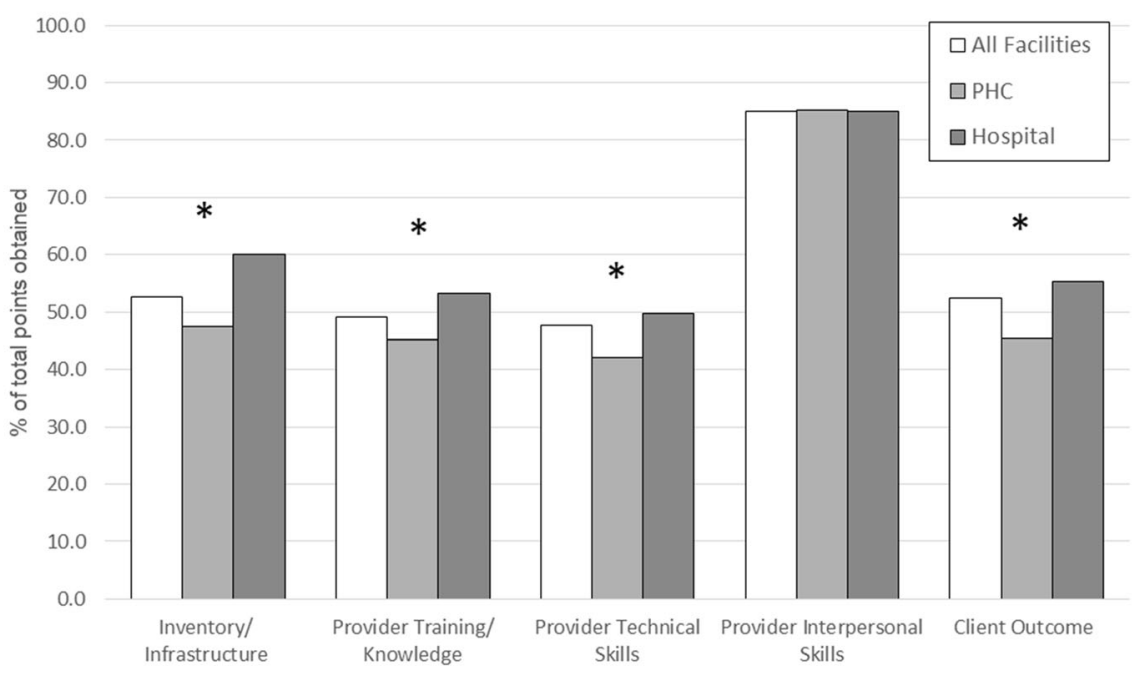

Fig. 2 Comparison of domains of quality of care across facility type. All domains except for interpersonal skills obtained a cumulative score of below $55 \%$. The lowest overall scores were obtained in provider and provider skill (47\%) and provider training/knowledge (49\%), while the highest scores were obtained in provider interpersonal skills (85\%). PHCs scored lower than hospitals in all domains except provider interpersonal skills 
Table 2 Characteristics of study clients and providers across facility type

\begin{tabular}{|c|c|c|c|c|}
\hline \multirow[t]{2}{*}{ Client characteristics } & \multirow{2}{*}{$\begin{array}{l}\text { Total } \\
(n=135)\end{array}$} & \multirow{2}{*}{$\begin{array}{l}\mathrm{PHC} \\
(n=38)\end{array}$} & \multirow{2}{*}{$\begin{array}{l}\text { Hospital } \\
(n=97)\end{array}$} & \multirow[t]{2}{*}{$P$-Value } \\
\hline & & & & \\
\hline$\overline{M e a n}$ age (years) $\pm S D$ & $27.87 \pm 8.23$ & $24.89 \pm 5.88$ & $28.32 \pm 5.15$ & 0.001 \\
\hline Mean gravidity \pm SD & $2.79 \pm 2.05$ & $3.16 \pm 2.49$ & $2.65 \pm 1.84$ & 0.256 \\
\hline Mean gestational age (weeks) $\pm S D$ & $28.26 \pm 8.43$ & $30.13 \pm 8.56$ & $27.43 \pm 8.29$ & 0.120 \\
\hline Mean gestational age at 1 st ANC visit (weeks) \pm SD & $20.83 \pm 7.78$ & $21.9 \pm 8.06$ & $20.49 \pm 7.71$ & 0.413 \\
\hline \multicolumn{5}{|l|}{ Mean \# ANC visits ${ }^{b}$} \\
\hline Gestational age $<20$ weeks $(n=13)$ & $2.23 \pm 1.79$ & $2.00 \pm 2.00$ & $2.33 \pm 1.80$ & 0.771 \\
\hline Gestational Age 20-29 weeks $(n=27)$ & $2.15 \pm 1.54$ & $2.60 \pm 1.52$ & $2.05 \pm 1.56$ & 0.477 \\
\hline Gestational age $30+$ weeks $(n=64)$ & $3.43 \pm 2.09$ & $3.39 \pm 1.9$ & $3.45 \pm 2.21$ & 0.920 \\
\hline \multicolumn{2}{|l|}{ Education [n (\%)] } & & & $<0.001$ \\
\hline Primary or below primary & $41(30.4)$ & $18(47.4)$ & $23(23.7)$ & \\
\hline Secondary & $41(30.1)$ & $19(50)$ & $22(22.7)$ & \\
\hline Postsecondary & $53(39.3)$ & $1(2.6)$ & $52(53.6)$ & \\
\hline \multicolumn{2}{|l|}{ Religion [n (\%)] } & & & 0.001 \\
\hline Christian & $60(44.4)$ & $8(21.1)$ & $52(53.6)$ & \\
\hline Muslim & $75(55.6)$ & $30(78.9)$ & $45(46.4)$ & \\
\hline \multicolumn{2}{|l|}{ Marital status [n (\%)] } & & & $1.000^{\circ}$ \\
\hline Married/living together & $130(96.3)$ & $37(97.4)$ & $93(95.9)$ & \\
\hline Divorced/separated/widowed & $5(3.7)$ & $1(2.6)$ & $4(4.1)$ & \\
\hline \multicolumn{2}{|l|}{ Health insurance coverage $[n(\%)]^{b}$} & & & 0.191 \\
\hline Yes & $19(23.8)$ & $2(11.8)$ & $17(27.0)$ & \\
\hline No & $61(76.3)$ & $15(88.2)$ & $46(73.0)$ & \\
\hline \multicolumn{2}{|l|}{ Socioeconomic status [n (\%)] } & & & 0.051 \\
\hline Low & $50(37.0)$ & $9(23.7)$ & $41(42.3)$ & \\
\hline Medium & $63(46.7)$ & $24(63.2)$ & $39(40.2)$ & \\
\hline High & $22(16.3)$ & $5(13.2)$ & $17(17.5)$ & \\
\hline \multicolumn{2}{|l|}{ Spending decision [n (\%)] ${ }^{\mathrm{b}}$} & & & 0.285 \\
\hline Myself & $10(8.3)$ & $3(8.6)$ & $7(8.2)$ & \\
\hline Partner & $49(40.8)$ & $16(45.7)$ & $33(38.8)$ & \\
\hline Both myself and partner & $51(42.5)$ & $11(31.4)$ & $40(47.1)$ & \\
\hline Other & $10(8.3)$ & $5(14.3)$ & $5(5.9)$ & \\
\hline \multirow[t]{2}{*}{ PROVIDERS } & Total & $\mathrm{PHC}$ & Hospital & \\
\hline & $(n=201)$ & $(n=105)$ & $(n=96)$ & \\
\hline Mean age \pm SD & $39.06 \pm 9.64$ & $38.15 \pm 9.79$ & $40.10 \pm 9.40$ & 0.164 \\
\hline \multicolumn{2}{|l|}{ gender $[n(\%)]$} & & & 0.396 \\
\hline Male & $37(18.4)$ & $17(16.2)$ & $20(20.8)$ & \\
\hline Female & $164(81.6)$ & $88(83.8)$ & $76(79.2)$ & \\
\hline \multicolumn{2}{|l|}{ Type of provider [n (\%)] } & & & $<0.001$ \\
\hline Obstetrician/Gynecologist & $6(3.0)$ & $0(0.0)$ & $6(6.3)$ & \\
\hline General Practitioner & $20(10.0)$ & $5(4.8)$ & $15(15.6)$ & \\
\hline Nurse/midwife & $83(41.3)$ & $19(18.1)$ & $64(66.7)$ & \\
\hline Community Health Extension Worker & $79(39.3)$ & $68(64.8)$ & $11(11.5)$ & \\
\hline Community Health Officer & $13(6.5)$ & $13(12.4)$ & $0(0.0)$ & \\
\hline
\end{tabular}


Table 3 Average Scores for Structural Attributes

\begin{tabular}{|c|c|c|c|c|c|c|c|c|c|c|}
\hline \multirow[t]{2}{*}{ Structure Attributes } & \multicolumn{3}{|l|}{ Total } & \multicolumn{3}{|c|}{ Primary } & \multicolumn{3}{|c|}{ Secondary } & \multirow[t]{2}{*}{$p$-value } \\
\hline & $\overline{\text { Mean }}$ & SD & $\%$ of total & $\overline{\text { Mean }}$ & SD & $\%$ of total & $\overline{\text { Mean }}$ & SD & $\%$ of total & \\
\hline Data collection tools (0-3) & 2.72 & 0.56 & 90.5 & 2.64 & 0.59 & 88.1 & 2.82 & 0.51 & 94.0 & $0.059^{*}$ \\
\hline Infrastructure (0-6) & 2.78 & 0.18 & 46.3 & 2.45 & 0.21 & 40.8 & 3.26 & 0.29 & 54.3 & 0.012 \\
\hline Guidelines (0-5) & 1.69 & 0.20 & 33.9 & 1.45 & 0.25 & 28.9 & 2.05 & 0.33 & 41.0 & 0.071 \\
\hline General equipment (0-11) & 6.43 & 0.30 & 58.5 & 5.96 & 0.40 & 54.2 & 7.10 & 0.43 & 64.6 & 0.030 \\
\hline Drugs (0-5) & 1.93 & 0.12 & 38.5 & 1.79 & 0.14 & 35.7 & 2.13 & 0.22 & 42.6 & 0.087 \\
\hline Referral mechanisms (0-4) & 2.40 & 0.11 & 60.0 & 2.20 & 0.14 & 54.9 & 2.69 & 0.18 & 67.3 & 0.016 \\
\hline Capacity for HDP-specific services (0-4) & 2.04 & 0.14 & 51.1 & 1.55 & 0.16 & 38.8 & 2.74 & 0.19 & 68.6 & 0.000 \\
\hline Total for inventory/ infrastructure (0-38) & 19.99 & 0.73 & 52.6 & 18.04 & 0.91 & 47.5 & 22.79 & 1.06 & 60.0 & 0.001 \\
\hline Training in last 3 years $(0-6)$ & 1.18 & 0.10 & 19.7 & 1.13 & 0.13 & 18.8 & 1.24 & 0.17 & 20.7 & 0.293 \\
\hline Signs of HDPs (0-12) & 7.66 & 0.22 & 63.8 & 7.21 & 0.32 & 60.1 & 8.14 & 0.30 & 67.8 & 0.019 \\
\hline Management of pre-eclampsia (0-8) & 4.15 & 0.18 & 51.8 & 4.22 & 0.25 & 52.8 & 4.06 & 0.26 & 50.8 & 0.669 \\
\hline Management of severe pre-eclampsia (0-9) & 4.85 & 0.24 & 53.9 & 4.42 & 0.35 & 49.2 & 5.31 & 0.33 & 59.0 & $0.032^{*}$ \\
\hline Management of eclampsia (0-11) & 6.29 & 0.32 & 57.1 & 5.47 & 0.46 & 49.7 & 7.17 & 0.44 & 65.2 & 0.004 \\
\hline Diagnosis of HDPs $(0-3)$ & 1.97 & 0.07 & 65.5 & 1.74 & 0.10 & 58.0 & 2.21 & 0.09 & 73.6 & 0.000 \\
\hline Usage of MgSO4 (0-7) & 1.63 & 0.12 & 23.2 & 1.12 & 0.13 & 15.9 & 2.18 & 0.20 & 31.1 & $0.000^{*}$ \\
\hline Usage of eclampsia prophylaxis (0-4) & 3.81 & 0.04 & 95.1 & 3.89 & 0.05 & 97.4 & 3.71 & 0.06 & 92.7 & $0.994^{*}$ \\
\hline Usage of antihypertensive drugs (0-5) & 0.92 & 0.08 & 18.4 & 0.68 & 0.09 & 13.7 & 1.18 & 0.13 & 23.5 & $0.001^{*}$ \\
\hline Total knowledge/ training score $(0-65)$ & 32.44 & 0.92 & 49.9 & 29.88 & 1.25 & 46.0 & 35.19 & 1.31 & 54.1 & 0.002 \\
\hline
\end{tabular}

*equal variances not assumed (using F statistic, $p<0.05$ )

\section{Determinants of Provider Knowledge}

\begin{tabular}{|c|c|}
\hline \multirow[b]{2}{*}{ Factors } & \multirow{2}{*}{$\begin{array}{l}\text { Beta } \\
\text { Coefficient }(95 \% \mathrm{Cl})\end{array}$} \\
\hline & \\
\hline Facility Type & $-112(-6.87-464)$ \\
\hline$(r e f=P H C)$ & \\
\hline Age (per year) & $0.22(-0.01,0.45)$ \\
\hline Gender & $-1.77(-7.84,4.28)$ \\
\hline $\begin{array}{l}\text { (ref=male) } \\
\text { General Practitioner } \\
\text { (ref=Ob/Gyn) }\end{array}$ & $-12.02(-18.90,-5.15)$ \\
\hline $\begin{array}{l}\text { Nurse/Midwife } \\
\text { (ref=Ob/Gyn) }\end{array}$ & $-15.41(-23.37,-7.45)$ \\
\hline $\begin{array}{l}\text { CHEW } \\
\text { (ref=Ob/Gyn) }\end{array}$ & $-23.80(-31.97,-15.63)$ \\
\hline $\begin{array}{l}\mathrm{CHO} \\
\text { (ref=Ob/Gyn) }\end{array}$ & $-18.49(-28.20,-8.79)$ \\
\hline Time Working at Facility (per year) & $-0.13(-0.36,0.10)$ \\
\hline-35 & 0 \\
\hline
\end{tabular}

Fig. 3 Linear regression coefficients examining provider knowledge score. Each row represents a variable included in the multivariable linear regression assessing factors associated provider knowledge scores. After adjustment for provider sociodemographic factors, the level of facility (primary vs. secondary) was no longer significant. Compared to obstetricians/gynecologists (reference group), each of the other provider types (general practitioner, nurse/midwife, $\mathrm{CHEW}$, and $\mathrm{CHO}$ ) obtained significantly lower knowledge scores 
Table 4 Average Scores for Process Attributes

\begin{tabular}{|c|c|c|c|c|c|c|c|c|c|c|}
\hline \multirow[t]{2}{*}{ Process Attribute } & \multicolumn{3}{|l|}{ Total } & \multicolumn{3}{|c|}{ Primary } & \multicolumn{3}{|c|}{ Secondary } & \multirow[t]{2}{*}{$p$-value } \\
\hline & $\overline{\text { Mean }}$ & SD & $\%$ of total & $\overline{\text { Mean }}$ & SD & $\%$ of total & $\overline{\text { Mean }}$ & SD & $\%$ of total & \\
\hline History taking for women with previous pregnancy (0-6) & 3.22 & 0.22 & 53.7 & 2.38 & 0.45 & 39.6 & 3.53 & 0.25 & 58.8 & 0.011 \\
\hline History taking (0-9) & 5.00 & 0.23 & 55.6 & 3.79 & 0.49 & 42.2 & 5.49 & 0.24 & 61.0 & 0.000 \\
\hline Physical exam (0-9) & 5.64 & 0.13 & 62.6 & 5.62 & 0.18 & 62.4 & 5.65 & 0.16 & 62.7 & 0.457 \\
\hline Lab tests $(0-2)$ & 1.04 & 0.08 & 52.2 & 0.85 & 0.16 & 42.3 & 1.13 & 0.09 & 56.3 & 0.060 \\
\hline Documentation (0-2) & 1.46 & 0.06 & 73.0 & 1.31 & 0.12 & 65.4 & 1.52 & 0.07 & 76.0 & 0.063 \\
\hline Disease prevention (0-12) & 8.35 & 0.21 & 69.6 & 8.47 & 0.34 & 70.6 & 8.30 & 0.26 & 69.2 & 0.644 \\
\hline Follow-up/continuation of care (0-3) & 2.35 & 0.08 & 78.3 & 2.44 & 0.12 & 81.2 & 2.31 & 0.10 & 77.1 & 0.752 \\
\hline Total provider/technical skills score $(0-37)^{a}$ & 17.64 & 0.44 & 47.7 & 15.61 & 0.84 & 42.2 & 18.43 & 0.49 & 49.8 & 0.002 \\
\hline Rapport (0-7) & 6.21 & 0.10 & 88.7 & 6.23 & 0.16 & 89.0 & 6.20 & 0.13 & 88.5 & 0.559 \\
\hline Communication (0-4) & 3.15 & 0.07 & 53.7 & 3.15 & 0.11 & 78.8 & 3.15 & 0.08 & 78.6 & 0.522 \\
\hline Total interpersonal skills score $(0-11)$ & 9.36 & 0.137 & 85.1 & 9.38 & 0.21 & 85.3 & 9.34 & 0.17 & 84.9 & 0.553 \\
\hline
\end{tabular}

a excluding history for women with previous pregnancy, as this category is relevant only for subset of population

the reference (obstetricians/gynecologists), it was found that general practitioners, nurses/midwives, CHEWs, and CHOs scored lower by 12.0 points $(\mathrm{p}=0.001), 15.4$ points $(p=0.000), 23.8$ points $(\mathrm{p}=0.000)$ and 18.5 points $(\mathrm{p}=0.000)$, respectively. Further adjustment for clustering by specific facility did not quantitatively or qualitatively change the results of the regression analysis.

\section{Process attributes of care: provider technical skills}

Table 4 shows the process attributes related to provider technical skills. Out of a total of 37 points, PHCs scored significantly lower than hospitals $(42.2 \%$ vs. $49.8 \%, p=0.0017)$. There were also significant differences between facility type for skills in general history taking $(42.2 \%$ vs. $61.0 \% ; p<0.001)$ and history taking for women with a previous pregnancy $(39.6 \%$ vs. $58.8 \%$; $p=0.0073)$. There were no significant differences in scores for physical examinations, lab tests, documentation, health promotion/prevention of disease, or encouragement of follow-up, but all categories except for documentation and encouragement of follow-up scored below $70 \%$ of potential points.

\section{Process attributes of care: provider interpersonal skills}

Table 4 also shows the process attributes related to provider interpersonal skills. Out of a total score of
11, PHCs and hospitals scored highly - 85.3 and $84.9 \%$, respectively. There were no significant differences observed in the scores of interpersonal subcategories, or in the cumulative interpersonal score between PHC and hospital care providers. However, individual indicators within the rapport-building and communications sub-categories were found to significantly differ (see Additional file 1). For example, PHCs more often used the client's name $(97.5 \%$ vs. $89.5 \%$; $p=0.016)$ and greeted in a friendly manner $(100 \%$ vs. $85.4 \% ; p=0.011$ ), but were less likely to maintain audio privacy $(67.5 \%$ vs. $92.6 \% ; p<0.001)$ and visual privacy $(65.0 \%$ vs. $90.5 \%$; $<<0.001)$, or inquire for need of other services $(35.9 \%$ vs. $83.3 \% ; p=0.022)$.

\section{Outcome attributes of care}

Table 5 shows the outcome attributes related to client experiences. Out of a total of 16, PHCs scored significantly lower than hospitals $(45.4 \%$ vs. $55.2 \% ; p=0.004)$. While perceptions of wait times, costs, and satisfaction were not significantly different between facility type, clients at PHCs had significantly lower health literacy than those at hospital health facilities $(39.5 \%$ vs. $50.6 \%$, $p=0.020$ ). Wait-time and time spent with providers were comparable between primary and secondary facilities, but women attending ANC at hospitals reported willingness

Table 5 Average Scores for Outcome Attributes

\begin{tabular}{|c|c|c|c|c|c|c|c|c|c|c|}
\hline \multirow[t]{2}{*}{ Outcome Attribute } & \multicolumn{3}{|l|}{ Total } & \multicolumn{3}{|c|}{ Primary } & \multicolumn{3}{|c|}{ Secondary } & \multirow{2}{*}{$\begin{array}{l}p^{-} \\
\text {value }\end{array}$} \\
\hline & mean & SD & $\%$ of total & mean & SD & $\%$ of total & mean & SD & $\%$ of total & \\
\hline Wait and cost (0-3) & 1.58 & 0.09 & 52.6 & 1.34 & 0.17 & 44.7 & 1.67 & 0.11 & 55.7 & 0.052 \\
\hline Satisfaction (0-5) & 3.01 & 0.11 & 60.3 & 2.76 & 0.23 & 55.3 & 3.11 & 0.12 & 62.3 & $0.092^{*}$ \\
\hline Health literacy (0-8) & 3.80 & 0.20 & 47.5 & 3.16 & 0.33 & 39.5 & 4.05 & 0.24 & 50.6 & 0.020 \\
\hline Total outcome score (0-16) & 8.39 & 0.26 & 52.5 & 7.26 & 0.51 & 45.4 & 8.84 & 0.30 & 55.2 & 0.004 \\
\hline
\end{tabular}


Table 6 Categorical and Continuous Outcomes

\begin{tabular}{|c|c|c|c|c|}
\hline Key outcome measures of quality of care & $\begin{array}{l}\text { Total } \\
(n=135)\end{array}$ & $\begin{array}{l}\text { PHC } \\
(n=38)\end{array}$ & $\begin{array}{l}\text { Hospital } \\
(n=97)\end{array}$ & $\begin{array}{l}p^{-} \\
\text {value }\end{array}$ \\
\hline$\overline{\text { Mean wait time (minutes) } \pm S D}$ & $93.8 \pm 78.13$ & $96.41 \pm 81.0$ & $92.85 \pm 77.63$ & 0.839 \\
\hline Wait time $[n(\%)]^{c}$ & & & & 0.285 \\
\hline$<1 \mathrm{~h}$ & $33(31.4)$ & $10(37.0)$ & $23(29.5)$ & \\
\hline $1-2 h$ & $45(42.9)$ & $8(29.6)$ & $37(47.4)$ & \\
\hline$>2 \mathrm{~h}$ & $27(25.7)$ & $9(33.3)$ & $18(23.1)$ & \\
\hline Mean time spent with provider (minutes) \pm SD & $20.4 \pm 17.68$ & $23.6 \pm 19.8$ & $18.8 \pm 16.7$ & 0.415 \\
\hline Time spent with provider $[\mathrm{n}(\%)]^{c}$ & & & & $0.185^{\mathrm{a}}$ \\
\hline$<5 \min$ & $9(21.4)$ & $1(7.1)$ & $8(28.6)$ & \\
\hline $5-19 \min$ & $15(35.7)$ & $7(50.0)$ & $8(28.6)$ & \\
\hline 20-39 min & $10(23.8)$ & $2(14.3)$ & $8(28.6)$ & \\
\hline$>40 \mathrm{~min}$ & $8(19.0)$ & $4(28.6)$ & $4(14.3)$ & \\
\hline First ANC visit $[n(\%)]^{c}$ & & & & $0.616^{\mathrm{a}}$ \\
\hline$<15$ weeks & $28(24.1)$ & $5(17.9)$ & $23(26.1)$ & \\
\hline 15-24 weeks & $41(35.3)$ & $9(32.1)$ & $32(36.4)$ & \\
\hline 25-30 weeks & $28(24.1)$ & $9(32.1)$ & 19 (21.6) & \\
\hline$>30$ weeks & $19(16.4)$ & $5(17.9)$ & $14(15.9)$ & \\
\hline Mean amount willing to pay for services (USD) ${ }^{b} \pm S D$ & $1.06 \pm 1.59$ & $0.43 \pm 0.40$ & $1.28 \pm 1.79$ & 0.044 \\
\hline Amount willing to pay for services $[n(\%)]^{c}$ & & & & $0.741^{a}$ \\
\hline$<0.27$ USD & $6(19.4)$ & $2(25.0)$ & $4(17.4)$ & \\
\hline $0.27-1.37$ USD & $18(58.1)$ & $5(62.5)$ & $13(56.5)$ & \\
\hline$>1.37$ USD & $7(22.6)$ & $1(12.5)$ & $6(26.1)$ & \\
\hline Satisfaction $[\mathrm{n}(\%)]^{\mathrm{c}}$ & & & & $0.110^{\mathrm{a}}$ \\
\hline Satisfied & $107(92.2)$ & $31(100.0)$ & $76(89.4)$ & \\
\hline Somewhat or not satisfied & $9(7.8)$ & $0(0.0)$ & $9(10.6)$ & \\
\hline Knowledge of danger signs in pregnancy [n (\%)] & & & & $0.504^{\mathrm{a}}$ \\
\hline 0 & $69(51.1)$ & $21(55.3)$ & $48(49.5)$ & \\
\hline 1 & $30(22.2)$ & $10(26.3)$ & $20(20.6)$ & \\
\hline 2 & $29(21.5)$ & $5(13.2)$ & $24(24.7)$ & \\
\hline 3 & $7(5.2)$ & $2(5.3)$ & $5(5.2)$ & \\
\hline
\end{tabular}

${ }^{a}$ using Fisher's exact test

${ }^{b} 1$ USD $=364.50 \mathrm{NGN}$

'effective sample size does not equal total sample due to missing data

to pay a higher amount for services (1.28 USD vs. 0.43 USD; $p=0.044$ ) (Table 6). More than half of all women $(51.1 \%)$ could not recall any danger signs in pregnancy that would compel them to return to a health provider. Only $5.2 \%$ of clients could name at least 3 danger signs most commonly severe headache, bleeding or fluids from vagina, and swelling of legs and feet (Table 6). Notably, satisfaction was very high for all facilities; $100 \%$ of respondents in PHCs and $89.4 \%$ of respondents in hospitals reported being satisfied with services received.

The difference observed in overall outcome score between PHCs and hospital facilities was no longer significant after regression adjustment for client age, level of education, and socioeconomic status $(p=0.189$, [Additional file 2]). Outcome scores for women with a "high" SES were $1.42(95 \% \mathrm{CI}=0.03,2.80)$ points lower than scores for women with a "low" SES $(p=0.045)$. Further adjustment for clustering by specific facility did not quantitatively or qualitatively change the results of the regression analysis.

\section{Discussion}

This analysis provides a comprehensive cross-sectional overview of the quality of ANC services provided across the six geo-political zones of Nigeria and explores how quality differs between PHC and hospitals. In general, it 
was found that the quality of ANC provided by Nigerian healthcare facilities is sub-par and does not meet many of the WHO-required elements for adequate ANC. All elements of quality of care, except for interpersonal skills, obtained a cumulative score of below 55\%. Furthermore, PHCs scored lower than hospitals in most domains. Despite these relatively low scores for quality of care, self-reports of client satisfaction were very positive.

\section{Structure}

Facilities captured in this study obtained very low scores for inventory and infrastructure - below half of the tools, recent guidelines, equipment, drugs, and mechanisms deemed necessary to perform antenatal services. Within general equipment, only three quarters of facilities had operational sphygmomanometers for measuring blood pressure, and less than half had dipsticks for proteinuria testing. These numbers are concerning, especially regarding the important role they play in diagnosing preeclampsia and eclampsia [22].

PHCs scored significantly lower than hospitals in almost all aspects of inventory and infrastructure. Ademiluyi and Aluko-Arowolo [23] have written on the unique infrastructural challenges faced by PHCs, citing Nigeria's colonial history and its implications on the evolution and distribution of healthcare resources as the root cause. To this day, the bulk of public healthcare dollars are spent in secondary facilities, as is government spending on general infrastructure [24]. While all levels of facilities must meet standards for accreditation, secondary level facilities have much stricter infrastructure requirements, including those for operating theatre, pharmacy, laboratory, and personnel capacities [25]. Despite their limited capacity to treat pregnant women, PHCs play a vital role in advising women on danger signs of obstetric complications, detecting these complications (including pre-eclampsia and eclampsia) early and referring women to general or specialist hospitals in a timely manner (often following initial stabilizing care, such as administration of loading dose of $\mathrm{MgSO}_{4}$ ) [26]. However, PHCs likely face challenges in efficiently getting patients to hospitals in the case of emergencies, as this study reveals that only a quarter have any ambulatory transport systems.

Other research has found a similar lack of infrastructure in both primary and secondary facilities in Nigeria. Kress et al. [12] reported on a "general shortage of drugs and supplies" that likely are the result of segmented supply chains and financial constraints placed on Local Government Authorities (LGAs) who are responsible for allocating resources to PHCs. They report that as much as $95 \%$ of funding must be allocated to staff salaries at the expense of adequate drugs, supplies, and maintenance. Oyekale [11] similarly found that tools and drugs essential for ANC including sphygmomanometers, thermometers, stethoscopes, magnesium sulfate, folic acid, and calcium gluconate were unavailable or dysfunctional in as many as $87 \%$ of facilities studied.

Provider knowledge and training received the lowest overall scores, with PHCs scoring significantly lower than hospitals. This finding is not entirely unexpected given the presence of lower level staff at PHCs compared to secondary facilities. Indeed, regression analysis shows that provider knowledge scores are dependent on the type of provider, (with obstetricians/gynecologists scoring at least 12 points higher than the next best cadre general practitioners). This is not to say that lower cadre health workers cannot be adequately trained on critical components of ANC, as has been demonstrated in previous research [27-30]. Despite existing evidence that shows task-shifting and training of lower-cadre healthcare workers is an important strategy for low-income countries, only $12.2 \%$ of providers in this study reported having received any training in the past 3 years.

\section{Process}

For both PHCs and hospitals, scores for providers' skills were moderate to low. Providers were relatively successful in the realm of 'follow-up/ continuation of care' (most clients were observed being instructed to come for a follow-up appointment and told where to go for follow-up). However, a smaller percentage were given a written reminder of when to come or encouraged to return in the case of emergency. It is well-documented that Nigerian women attend fewer ANC consultations than is recommended for healthy pregnancy and childbirth [8]. Providing written reminders may be a simple strategy to improve rates of ANC attendance. Previous research in Zanzibar has shown the effects of follow-up reminders (via SMS) to be beneficial in not only the number of ANC visits attended, but also in the percentage of women with antenatal complications identified and referred [31].

In 2015, Fagbamigbe and Idemudia [16] studied ANC quality in Nigeria and reported on the eight nationally recommended 'critical components' of ANC (blood pressure, iron supplements, blood sample, urine sample, tetanus injections, danger signs, HIV tests, malaria prophylaxis). As in our study, the most common of these eight components practiced were blood pressure measurement and provision of iron/folic acid supplements. Compared with those in Fagbamigbe and Idemudia, the participants in our study reported higher frequency of HIV testing (78.5\% vs. $41.7 \%)$ and provision of malaria prophylaxis $(77.8 \%$ vs. $40.1 \%)$, and lower frequency of urine testing $(28.1 \%$ vs. $81.9 \%)$. Furthermore, process scores may be an optimistic interpretation of the provider's skill in these areas; although the data tells us that 
the actions were taken, it does not specify the degree to which they were correctly completed. For example, there are many sources of inaccuracy for blood pressure measurement including patient, device, procedure, and observer-related sources [32]. Previous research has identified blood pressure inaccuracy as a challenge in hospitals in developing nations, and especially among providers with lower qualifications such as nurses and CHOs [33, 34].

Provider interpersonal skills, as assessed by direct observation of their interaction with clients, scored highly. Audio and visual privacy was the only interpersonal indicator in which PHCs performed worse than secondary facilities; this is understandable given the relatively little space and busy atmosphere of PHCs. Babatunde et al. [35] found similarly positive results among PHC users, with the ideal interpersonal skills being displayed in 71$91 \%$ of healthcare interactions.

\section{Outcome}

Low scores in health literacy (dissemination, communication, and explanation of consultation results) were reflected in clients' poor recall of danger signs of pregnancy. Hospitals did significantly better in this regard than PHC, possibly because of better-trained staff, or more educated clients. Gaining sufficient and adequate information about one's health and potential complications from a care provider is important during the antenatal period and is associated with improved ANC attendance and pregnancy outcomes [36-38]. Health literacy is critical to patient empowerment, and accurate information from an informed provider is especially valuable when the alternative might be informal education, misrepresentation, or mythology.

Despite the low scores received for structural and process attributes, as well as moderate perceptions of wait time and cost, all facilities scored extremely well in terms of client satisfaction. This result is encouraging, as each new interaction with the health system presents an additional opportunity for HDPs to be detected and managed. However, perception of care must be interpreted cautiously, as low-income and low-education women can be uncritical of the healthcare they receive or base it on the provider's interpersonal skills rather than their competence $[17,39,40]$. Only a quarter of clients attended an ANC visit before 15 weeks gestational age, despite the WHO's recommendations for an initial contact within 12 weeks of pregnancy. This is especially important for first time mothers, and to ensure early detection of HDPs, HIV, and anemia [1].

In 2016, the WHO released "Standards for Improving Quality of Maternal and Newborn Care in Health Facilities" a document which outlines 8 domains of quality of care that should be assessed, improved, and monitored within the health system [41]. These range from the appropriate use of data, to effective and compassionate communication with women and their families, to appropriate physical environment and competent, motivated staff. Furthermore, quality antenatal care should result in a "positive pregnancy experience", defined as not only maintaining a healthy pregnancy for the mother and baby, but "having an effective transition to positive labor and birth", and "achieving positive motherhood (including maternal self-esteem, competence and autonomy)" [1]. In accordance with these standards, our study shows that improvements to ANC must be holistic and encompass not just the facility, but also provider knowledge and practices. It must also involve community mobilization to educate on the importance of ANC and improve maternal health literacy. In an environment where resources are limited and there are competing interests in terms of health priorities, solutions must be innovative and easy to adopt, especially for PHCs. This may include mobile training and decision-making tools, such as the m4Change application created as part of the Nigerian government's Saving One Million Lives Initiative [29]. Policy that mandates regular training refreshers around ANC skills could help to close knowledge gaps. Improvements should be evidence-based and outcomesoriented, meaning that they are measured using outcome indicators such as the number of women who have at least four ANC visits, tetanus protection at birth, or the proportion of women with a written birth and emergency plan at 37 weeks of pregnancy [42]. This research comes during the development of a new National Health Act, in which the Federal Government of Nigeria has committed to "reactivate" and "revitalize" the country's PHCs. This has begun with the inauguration of an interagency Supply Chain Committee, which will act as the implementation partner for the $\mathrm{PHC}$ revitalization agenda [43]. This research may therefore serve as a framework for which major gaps in PHC quality, specifically as it pertains to ANC and the ability to manage HDPs, require immediate attention.

\section{Limitations}

There are several limitations to our approach in the identification of indicators that adequately assess quality of ANC, and more specifically its capacity to detect and manage HDPs, as there is little consensus or predetermined framework for doing so. Quality and abundance of indicators are further limited in that they only include aspects of care that should be provided at every ANC consultation (data is cross-sectional and does not encompass multiple ANC visits for a single patient). Indicators used for "outcome" are limited in that they do not capture postnatal outcomes such as birth success, incidence of disease, survival, or long-term health conditions. However, they do measure outcomes of the 
consultation itself by showing how much knowledge and satisfaction a woman gained from her ANC visit, and how likely she would be to return. Facilities and clients were selected purposively, leading to potential selection bias and lack of external generalizability. However, because it was conducted across the country (and across all geo-political zones) - it reflects the population of most women attending ANC in Nigerian facilities. As with all studies that rely on interview-based data collection, this study is limited by interviewer and respondent bias. To mitigate this, research assistants were trained on interviewing and data collection techniques. Additionally, interview sources were supplemented with more objective data sources - facility and ANC visit observational checklists.

\section{Conclusion}

We identified three major obstacles to quality ANC in Nigeria and its ability to detect and manage HDPs: 1) inadequate infrastructure, particularly in the lack of facility guidelines, drug stock, referral mechanisms, and general equipment including sphygmomanometers and urine dipsticks; 2) very inadequate provider knowledge, due to infrequent training updates and low understanding of the use of drugs to prevent and treat HDPs, and 3) poor communication of health results to clients, resulting in low maternal health literacy. These issues are generally exacerbated in PHCs vs. hospitals due to lower level cadres of healthcare workers and inadequacy of funding for essential supplies, equipment, and infrastructure. Future research may wish to explore additional elements of the quality of ANC in Nigeria and how it impacts birth and postnatal outcomes. This includes outcomes of eclampsia seizures, likelihood and success of cesarean section following PE/E indication, days of hospitalization, and admission to intensive care.

\section{Additional files}

Additional file 1: Table of the prevalence of each indicator listed under provider interpersonal skills and comparison of prevalence between PHCs and hospitals. (DOCX $13 \mathrm{~kb}$ )

Additional file 2: Linear regression table examining the effect of client demographic variables including age, SES, and education on client outcome scores. (DOCX $13 \mathrm{~kb}$ )

\section{Abbreviations}

ANC: antenatal care; BP: blood pressure; CHEW: Community Health Extension Worker; $\mathrm{CHO}$ : Community Health Officer; HDP: Hypertensive Disorders in Pregnancy; HIV: human immunodeficiency virus; HT: hypertension; LGA: Local Government Authorities; $\mathrm{MgSO}_{4}$ : magnesium sulfate; PE/E: pre-eclampsia/ eclampsia; PHC: primary healthcare center; PMTCT: prevention of mother to child transmission; QOC: quality of care; SES: socioeconomic status; STI: sexually transmitted infection; TB: tuberculosis; WHO: World Health Organization

\section{Acknowledgements}

We thank all clients and healthcare providers who participated in the landscape analysis, and staff at the affiliated facilities. We also thank the Ending Eclampsia team based in Abuja, Nigeria, comprised of Gloria Adoyi, Roli Akpolo, Emmanuel Nwala, and Charles Nwaigwe. Additionally, Dr. Pooja Sripad, Dr. Timothy Abuya, and Dr. George Eluwa (Population Council) provided statistical, methodological, and general support.

\section{Authors' contributions}

$\mathrm{CW}, \mathrm{SI}$, and KK contributed to development of the protocol, data collection, and reviewing the manuscript. AS was responsible for data cleaning, analysis, and writing the manuscript. All authors read and approved the final manuscript.

\section{Funding}

Support for this paper was made possible by the generous support of the American people through the United States Agency for International Development (USAID) under the terms of USAID AID-OAA-A-14-00048. The contents of this paper are the sole responsibility of the authors and do not necessarily reflect the views of USAID or the United States Government. Apart from requesting that the study cover Nigeria's six geo-political zones, the funding body did not play a role in specific site selection, design, execution, analysis or write-up of this study.

\section{Availability of data and materials}

The datasets from the current study are available from the corresponding author on reasonable request.

\section{Ethics approval and consent to participate}

Ethical approval was granted by the Population Council's Institutional Review Board (IRB) in New York City (p. 693), the National Health Research Ethics Committee (NHREC) of the Federal Ministry of Health (FMoH), Nigeria (NHREC/01/01/2007-20/06/2015b), and the ethics committees of all the participating states within Nigeria. Written, informed consent was obtained from each participant, with emphasis that the study was voluntary and confidential. Data collectors were trained on ethical conduct.

\section{Consent for publication}

Not applicable.

\section{Competing interests}

The authors declare that they have no competing interests.

\section{Author details}

'Dalla Lana School of Public Health, Toronto, Canada. ${ }^{2}$ Population Council, Abuja, Nigeria. ${ }^{3}$ Population Council, New York, USA. ${ }^{4}$ Population Council, Washington, USA.

Received: 13 April 2018 Accepted: 4 June 2019

Published online: 24 June 2019

\section{References}

1. World Health Organization. WHO recommendations on antenatal care for a positive pregnancy experience: World Health Organization; 2016.

2. Oladapo O, Adetoro O, Ekele B, Chama C, Etuk S, Aboyeji A, et al. When getting there is not enough: a nationwide cross-sectional study of 998 maternal deaths and 1451 near-misses in public tertiary hospitals in a lowincome country. BJOG. 2016;123(6):928-38.

3. Mammaro A, Carrara S, Cavaliere A, Ermito S, Dinatale A, Pappalardo EM, et al. Hypertensive disorders of pregnancy. J Perinat Med. 2009;3(1):1.

4. Oyerinde K. Can antenatal care result in significant maternal mortality reduction in developing countries. J Community Med Health Educ. 2013; $3(2): 2-3$.

5. WHO. World health statistics 2014. Geneva: World Health Organization; 2014

6. UNICEF. Antenatal Care - UNICEF data. https://data.unicef.org/topic/ maternal-health/antenatal-care/\#. Accessed 23 May 2017.

7. Omer K, Afi NJ, Adamu M, Malami SA, Oyo-Ita A, Cockcroft A, et al. Seeking evidence to support efforts to increase use of antenatal care: a crosssectional study in two states of Nigeria. BMC pregnancy childbirth. 2014; 14(1):380. 
8. Mallick L, Tukur D, Kerry M. Trends in maternal health in Nigeria, 2003-2013. DHS Further Analysis Reports. 2016; (102).

9. Donabedian A. Evaluating the quality of medical care. The Milbank memorial fund quarterly. 1966:44(3):166-206.

10. Ayanian JZ, Markel H. Donabedian's lasting framework for health Care quality. N Engl J Med. 2016;375(3):205-7.

11. Oyekale AS. Assessment of primary health care facilities' service readiness in Nigeria. BMC Health Serv Res. 2017;17(1):172.

12. Kress DH, Su Y, Wang H. Assessment of primary health Care system performance in Nigeria: using the primary health Care performance Indicator conceptual framework. Health Systems \& Reform. 2016;2(4):302-18.

13. Warren CE, Ishaku S, Oginni A, Adoyi G, Kirk KR, Dempsey A. Landscape analysis of pre-eclampsia/eclampsia in Nigeria. Abuja: Population Council; 2015.

14. Balogun O. Patients perception of quality of antenatal service in four selected private health facilities in Ilorin, Kwara state of Nigeria. Niger Med Pract. 2007;51(4):80-4.

15. Oladapo OT, Iyaniwura CA, Sule-Odu AO. Quality of antenatal services at the primary care level in Southwest Nigeria. Afr J Reprod Health. 2008;12(3):71-92.

16. Fagbamigbe AF, Idemudia ES. Assessment of quality of antenatal care services in Nigeria: evidence from a population-based survey. Reprod Health. 2015;12(1):88.

17. Warren CE, Abuya T, Kanya L, Obare F, Njuki R, Temmerman M, et al. A cross sectional comparison of postnatal care quality in facilities participating in a maternal health voucher program versus non-voucher facilities in Kenya. BMC pregnancy childbirth. 2015;15(1):153

18. Bruce J. Fundamental elements of the quality of care: a simple framework. Stud Fam Plan. 1990;21(2):61-91.

19. Hulton L, Matthews Z, Stones RW. Applying a framework for assessing the quality of maternal health services in urban India. Soc Sci Med. 2007;64(10): 2083-95.

20. Vyas S, Kumaranayake L. Constructing socio-economic status indices: how to use principal components analysis. Health Policy Plan. 2006;21 (6):459-68.

21. StataCorp. 2017. STATA (release 15) statistical software. College Station, TX: StataCorp LLC

22. WHO. WHO recommendations for prevention and treatment of preeclampsia and eclampsia. Geneva: World Health Organization; 2011.

23. Ademiluyi IA, Aluko-Arowolo SO. Infrastructural distribution of healthcare services in Nigeria: an overview. J Geog Reg Plann. 2009;2(5):104-10.

24. Central Bank of Nigeria. Annuals report and statement of account for the year ended 31st December 2005. https://www.cbn.gov.ng/OUT/2010/ PUBLICATIONS/REPORTS/RSD/Link\%20Files/INTERACTIVE\%2OANNUAL \%20REPORT\%20FOR\%20THE\%20YEAR\%20ENDED\%20DECEMBER\%202009. pdf. Accessed 17 Aug 2017

25. National Health Insurance Scheme. National Health Insurance Scheme Operational Guidelines. http://www.dhmlnigeria.com/downloads/NHIS OPERATIONAL_GUIDELINES(Revised).pdf. Accessed 17 Aug 2017.

26. Oguntunde O, Charyeva Z, Cannon M, Sambisa W, Orobaton N, Kabo IA, et al. Factors influencing the use of magnesium sulphate in pre-eclampsia/ eclampsia management in health facilities in northern Nigeria: a mixed methods study. BMC Pregnancy Childbirth. 2015;15(1):130.

27. Fulton BD, Scheffler RM, Sparkes SP, Auh EY, Vuijicic M, Soucat A. Health workforce skill mix and task shifting in low income countries: a review of recent evidence. Hum Resour Health. 2011;9(1):1.

28. Ishaku S, Ahonsi B, Tukur J, Ayodeji O. Attrition from care after the critical phase of severe pre-eclampsia and eclampsia: insights from an intervention with magnesium sulphate in a primary care setting in northern Nigeria. Health. 2013:5(09):1461.

29. McNabb M, Chukwu E, Ojo O, Shekhar N, Gill CJ, Salami H, et al. Assessment of the quality of antenatal care services provided by health workers using a mobile phone decision support application in northern Nigeria: a pre/postintervention study. PLoS One. 2015:10(5):e0123940.

30. Ameh CA, Kerr R, Madaj B, Mdegela M, Kana T, Jones S, et al. Knowledge and skills of healthcare providers in sub-Saharan Africa and Asia before and after competency-based training in emergency obstetric and early newborn care. PLoS One. 2016;11(12):e0167270

31. Lund S, Nielsen BB, Hemed M, Boas IM, Said A, Said K, et al. Mobile phones improve antenatal care attendance in Zanzibar: a cluster randomized controlled trial. BMC Pregnancy Childbirth. 2014;14(1):29.

32. Kallioinen N, Hill A, Horswill MS, Ward HE, Watson MO. Sources of inaccuracy in the measurement of adult patients' resting blood pressure in clinical settings: a systematic review. J Hypertens Manag. 2017;35(3):421.
33. Ahmed MEBK. Knowledge of blood pressure measurement among a teaching hospital staff in a developing nation. J Hum Hypertens. 1997; 11(8):495.

34. Ayodele O, Okunola O, Akintunde A, Sanya E. End digit preference in blood pressure measurement in a hypertension specialty clinic in Southwest Nigeria: cardiovascular topics. Cardiovasc J Afr. 2012;23(2):85-9.

35. Babatunde OA, Aiyenigba E, Awoyemi OA, Akande TM, Musa Ol, Salaudeen AG, et al. Primary health Care Consumers' perception of quality of Care and its determinants in north-Central Nigeria. J Asian Sci Res. 2013;3(7):775.

36. Kohan S, Ghasemi S, Dodangeh M. Associations between maternal health literacy and prenatal care and pregnancy outcome. Iran J Nurs Midwifery Res. 2008; 12:4

37. Simkhada $B$, van Teijlingen ER, Porter M, Simkhada P. Factors affecting the utilization of antenatal care in developing countries: systematic review of the literature. J Adv Nurs. 2008;61(3):244-60.

38. Mojoyinola J. Influence of maternal health literacy on healthy pregnancy and pregnancy outcomes of women attending public hospitals in Ibadan, Oyo state, Nigeria. Afr Res Rev. 2011;5:3.

39. Porter $M$, Macintyre $S$. What is, must be best: a research note on conservative or deferential responses to antenatal care provision. Soc Sci Med. 1984;19(11):1197-200.

40. Fawole AO, Okunlola MA, Adekunle AO. Clients' perceptions of the quality of antenatal care. J Natl Med Assoc. 2008;100(9):1052-8.

41. WHO. Standards for improving quality of maternal and newborn care in health facilities. Geneva: World Health Organization; 2016.

42. Lincetto O, Mothebesoane-Anoh S, Gomez P, Munjanja S. Antenatal care. In: Lawn J, Kerber K, editors. Opportunities for Africa's newborns: practical data, policy and programmatic support for newborn care in Africa. Capetown: Mills Lotho; 2006. p. 55-62.

43. Stewart S, Charles J. Nigeria's President Commits to Revitalizing 10,000 Primary Health Care Centers. North Bethesda; USAID; 2017.

\section{Publisher's Note}

Springer Nature remains neutral with regard to jurisdictional claims in published maps and institutional affiliations.

Ready to submit your research? Choose BMC and benefit from:

- fast, convenient online submission

- thorough peer review by experienced researchers in your field

- rapid publication on acceptance

- support for research data, including large and complex data types

- gold Open Access which fosters wider collaboration and increased citations

- maximum visibility for your research: over $100 \mathrm{M}$ website views per year

At $\mathrm{BMC}$, research is always in progress.

Learn more biomedcentral.com/submissions 